\title{
Serine-to-alanine substitutions at the amino-terminal region of phytochrome A result in an increase in biological activity
}

\author{
Jörg Stockhaus, ${ }^{1-3}$ Akira Nagatani, ${ }^{2}$ Ursula Halfter, ${ }^{1,2}$ Steve Kay, ${ }^{1,4}$ Masaki Furuya, ${ }^{2,5}$ \\ and Nam-Hai Chua ${ }^{1,6}$ \\ ${ }^{1}$ The Rockefeller University, Laboratory of Plant Molecular Biology, New York, New York 10021-6399 USA; ${ }^{2}$ Frontier \\ Research Program, Laboratory of Plant Biological Regulation, RIKEN Institute, Wako, Saitama, 351-01, Japan
}

\begin{abstract}
We have used a tobacco transgenic plant system to assay the structure/function relationship of phytochrome A (phyA), a plant photoreceptor. The amino terminus of phyA from different plant species is very rich in serine residues. To investigate whether these serine residues are required for phytochrome function, the first 10 serine codons encoding amino acid residues $2-4,10-14,19$, and 20 in the amino-terminal domain of the rice phyA gene (phyA) were changed to alanine codons. The mutant (S/A phyA), as well as the wild-type phy $A$ cDNA, was placed under the control of the $35 S$ promoter, and the chimeric genes were transferred into the tobacco genome by Agrobacterium-mediated transformation. Transgenic tobacco plants expressing either wild-type or S/A phyA showed similar phenotypic alterations, including dwarfism and dark-green leaves. However, hypocotyl elongation experiments revealed that transgenic seedlings expressing S/A phyA showed a higher amplitude of the red light response with respect to the inhibition of hypocotyl elongation. The observed difference is not correlated with expression levels of the transgene. The chromophore is attached to the mutant phyA apoprotein (PHY A), and the mutant photoreceptor is photoreversible, giving a difference spectrum indistinguishable from that of the rice phyA. Our results indicate that the $S / A$ mutant has a higher biological activity as compared with the wild-type rice phy $A$.
\end{abstract}

[Key Words: Phytochrome; structure/function; tobacco]

Received August 7, 1992; revised version accepted October 1, 1992.

Autotrophic plants depend on light as the primary energy source. For this reason, it is essential for the plant to continuously monitor and adapt to ambient light conditions. The light-sensing function is carried out by several photosensory pigments that are able to measure light quantity and quality. In addition, the light-detection mechanism is used to trigger various plant developmental processes ranging from germination to flowering (Kendrick and Kronenberg 1986; Smith and Whitelam 1990). One of these developmental processes is a phenomenon termed photomorphogenesis. When germinated in complete darkness, higher plant seedlings exhibit certain characteristic features, including elongated internodes, reduced leaf size, no chlorophyll synthesis, and arrested development. Upon exposure to light these eti-

Present addresses: ${ }^{3}$ Institut für Entwicklungs- und Molekularbiologie der Pflanzen, Heinrich-Heine Universität Düsseldorf, 4000 Düsseldorf 1 , Germany; ${ }^{4}$ Department of Biology, University of Virginia, Charlottesville, Virginia 22901 USA; ${ }^{5}$ Advanced Research Laboratory, Hitachi, Ltd. Laboratory of Plant Biological Regulation, Hatoyama, Saitama, Japan 350-03.

${ }^{6}$ Corresponding author. olated seedlings undergo photomorphogenesis that is characterized by chlorophyll synthesis, chloroplast development, leaf expansion, and the cessation of rapid internode elongation. It is known that this process is regulated by the complex interaction of several different photoreceptors (Furuya 1987; Thomas and Johnson 1991). One of these photoreceptors is red light-absorbing phytochrome, which is the best characterized plant photoreceptor to date (Furuya 1989; Smith and Whitelam 1990). Phytochrome is a chromoprotein consisting of an apoprotein (molecular mass of monomer, 118-125 kD) covalently linked to a linear tetrapyrrole /Lagarias and Rapoport 1980). It is synthesized in the dark as the red light-absorbing form ( $\operatorname{Pr}_{i} \lambda \max .=666 \mathrm{~nm}$ ), which is physiologically inactive. Absorption of red light converts the Pr form to the biologically active far red-absorbing form $(\mathrm{Pfr} ; \lambda \max .=730 \mathrm{~nm}$ ). The light-induced conversion between the Pr and Pfr forms is photoreversible. Phytochrome therefore serves as a molecular light switch, being activated by red light and attenuated by far red light.

Several cDNA and genomic clones encoding phy- 
tochrome have been isolated and characterized (for review, see Quail 1991). Sequence analysis revealed that the cDNA clones encode distinct apoproteins (PHY A, PHY B, PHY C, etc.). Among these different types of phytochrome, phytochrome A (phyA), also referred to as type I phytochrome (Furuya 1989), is the best characterized so far. Some data have accumulated concerning its domain structure and the conformational changes that the molecule undergoes during photoconversion (Vierstra and Quail 1986; Yamamoto 1990; Parker et al. 1991). However, very little is known about the sequence of molecular events by which phyA triggers the diverse physiological responses. The availability of full-length cDNA clones encoding phyA from different plant species made it possible to express this particular photoreceptor in transgenic plants. It was found that expression of monocot phyA in transgenic tobacco or tomato plants induces phenotypic changes that include semidwarfism and dark-green leaves (Boylan and Quail 1989; Keller et al. 1989; Nagatani et al. 1991), as well as altered patterns of endogenous $c a b$ gene expression (Kay et al. 1989b). In particular, it was shown that the introduced rice gene phy $A$ can regulate the hypocotyl length of transgenic tobacco seedlings, resulting in shorter hypocotyls, as compared with untransformed control seedlings, under dim white light (Nagatani et al. 1991). Similar results were obtained with the expression of oat phyA in transgenic Arabidopsis (Boylan and Quail 1991). Therefore, these transgenic systems can be used to analyze the structure/function relationship of phyA in vivo.

We are interested in studying the functions of different domains of phyA using rice phyA as a model system. We have first focused on the amino-terminal domain, because previous studies (Vierstra and Quail 1986; Yamamoto 1990; Parker et al. 1991) demonstrated that this domain (amino-terminal $10 \mathrm{kD}$ of phytochrome) undergoes significant conformational changes upon photoconversion from the Pr to the Pfr form /Cordonnier et al. 1985; Jones et al. 1985; Lagarias and Mercurio 1985; Chai et al. 1987; Vierstra et al. 1987). In the Pfr form, the amino-terminal domain interacts with the chromophore environment and alters the spectral properties of this molecule (Vierstra and Quail 1983). An inspection of amino-terminal amino acid sequences of phyA from different species revealed that these sequences are enriched in serine residues, which are potential target sites for phosphorylation. It has been demonstrated that at least one of the serine residues can be phosphorylated in vitro by a polycation-stimulated protein kinase activity that is associated with purified phytochrome (Wong et al. 1986). These observations prompted us to examine whether the 10 amino-terminal serine residues of rice phyA are necessary for its function. We show here that substitution of these serine residues by alanine residues resulted in a phyA mutant with a higher biological activity.

\section{Results}

Expression of mutated rice phyA $C D N A$ in tobacco

The amino acid sequences of the amino-terminal region of phyA from different species contain a preponderance of serine residues. Figure 1A compares the first 20 amino acids of phyA from different monocotyledonous and dicotyledonous species. The serine-rich character is conserved among phyA molecules from evolutionarily divergent species such as the monocot rice and the dicot tobacco (Fig. 1A). Of the first 20 amino-terminal amino acids of rice phyA, 10 are serine residues (amino acids $2-4,10-14,19$, and 20). This observation prompted us to investigate whether these serine residues are essential for the function of rice phyA in vivo. To address this question, we have chosen to assay the activity of mutated phyA in transgenic tobacco plants. The first 10 serine codons of a rice phy A cDNA (Kay et al. 1989a) were changed to alanine codons (Fig. 1B), and the mutated cDNA was fused downstream of the $35 \mathrm{~S}$ promoter of cauliflower mosaic virus. This construct was transferred into the Nicotiana tabacum var. Xanthi genome by Agrobacterium-mediated transformation. Transgenic tobacco plants expressing rice wild-type (RW) phyA under the transcriptional regulation of the $35 \mathrm{~S}$ promoter were used as controls throughout these studies.

Eight independent transgenic plants expressing the mutated rice phyA cDNA (S/A) showed phenotypic changes, such as shortened internodes and dark-green leaves. Similar phenotypes have been described previously for plants expressing wild-type phy $A$ genes (Boylan and Quail 1989; Keller et al. 1989; Nagatani et al. 1991). This characteristic phenotype indicated that the rice S/A phyA mutant was still functional (Fig. 1C). Because the plants shown in Figure 1C were grown under light conditions that were saturating for most phytochrome responses, it was not possible to perform comparative studies of transgenic plants expressing either the rice wild-type phyA (RW phyA) or the S/A phyA mutant. To analyze the biological activity of the expressed rice phyA molecules, we have chosen the hypocotyl elongation assay described by Nagatani et al. (1991) as a sensitive experimental system.

$T_{1}$ seeds of independent transgenic plants were imbibed under white light for $24 \mathrm{hr}$ and subsequently irradiated with $15 \times 10^{-3} \mathrm{~W} / \mathrm{m}^{2}$ red light for 6 days. Under these growth conditions, phytochrome is limiting for the inhibition of hypocotyl elongation, which is known to be a characteristic phytochrome response. Figure 1D shows representative seedlings grown on the same plate under the conditions described above. The hypocotyl of the untransformed Xanthi seedlings was elongated. This elongation was inhibited in seedlings expressing RW phyA (RW1) and was further suppressed in transgenic seedlings expressing S/A phyA (S/A2). This observation indicates that the biological activity of S/A phyA might be higher than that of RW phyA. To test whether this is a general phenomenon, the hypocotyl length of $60 \mathrm{~T}_{1}$ seedlings for each of the six RW and S/A lines was determined. Figure 2A shows that the seedlings can be separated into two populations based on hypocotyl length. There was a cosegregation of the short hypocotyl phenotype, with kanamycin resistance conferred by the transgene. The stunted S/A population (maximum hypocotyl length $=$ 


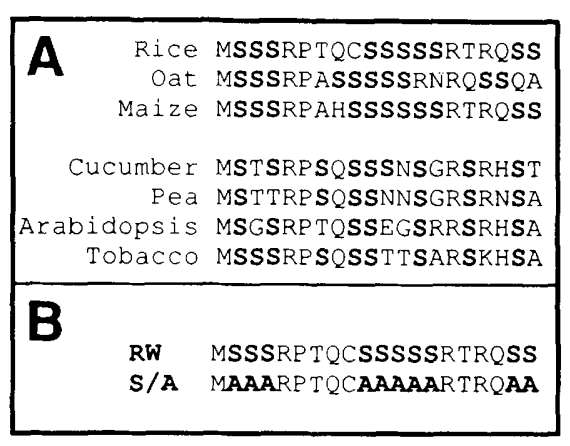

Figure 1. (A) Representation of the 20 amino-terminal amino acids from PHY A of three monocotyledonous plants, rice (Kay et al. 1989a), oat (Hershey et al. 1985), and maize (Christensen and Quail 1989), and four dicotyledonous plants, zucchini (Sharrock et al. 1986), pea (Sato 1988), Arabidopsis (Sharrock and Quail 1989), and tobacco (M. Deak, R. Kern, S. Kay, unpubl.) $(B)$ Amino-terminal 20 amino acids of the RW phyA and the $\mathrm{Ser} \rightarrow \mathrm{Ala}$ mutant (S/A). All of the 10 serine residues were substituted by alanine residues. $(C)$ Tobacco plants grown in soil for 10 weeks under saturating white light. (XAN) Tobacco wild-type var. Xanthi; (S/A2) Xanthi line expressing the rice phyA mutant; (RWI) Xanthi line expressing RW phyA. (D) Seven-day-old tobacco seedlings after irradiation with $1.2 \times 10^{-3} \mathrm{~W} / \mathrm{m}^{2}$ continuous red light. (XAN) Tobacco wild-type var. Xanthi; (S/A) Xanthi line expressing S/A phyA; (RW1) Xanthi line expressing RW phyA.
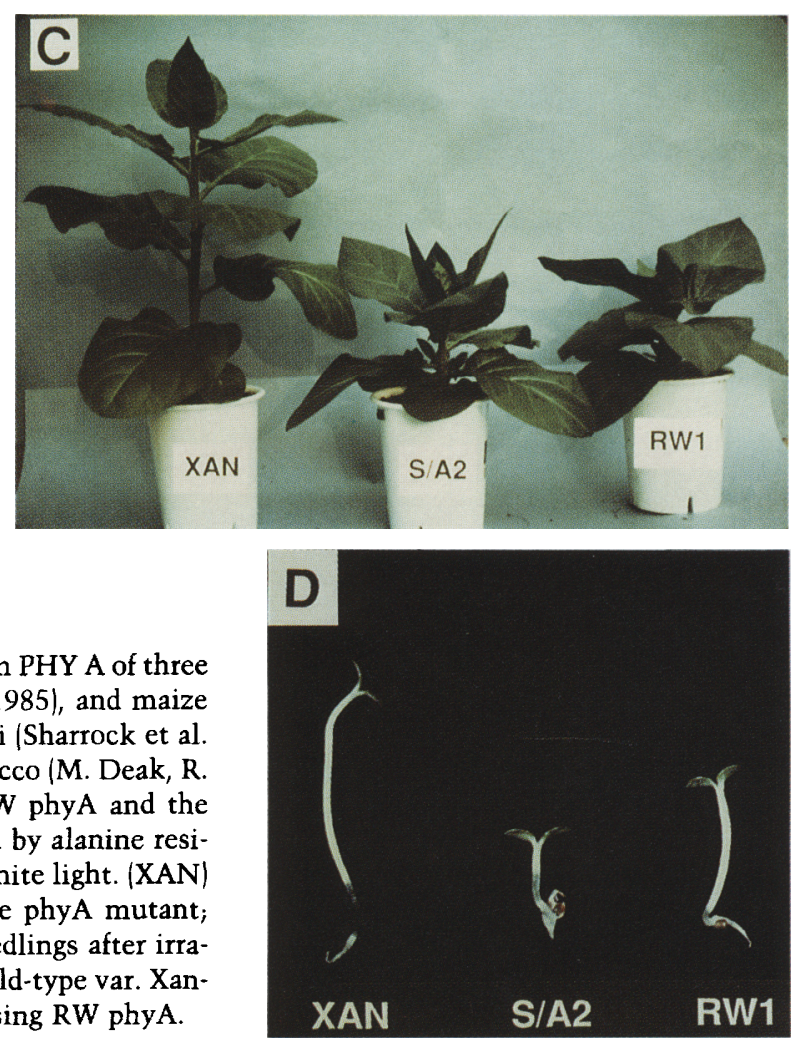

$4 \mathrm{~mm} /$ is $\sim 2-3 \mathrm{~mm}$ shorter than the corresponding RW population (maximum hypocotyl length $=7 \mathrm{~mm}$ ). This difference in hypocotyl length was consistently observed for all transgenic RW and S/A lines analyzed to date.

To test whether the rice phytochrome expression has any nonspecific inhibitory effect on hypocotyl elongation, the hypocotyl lengths of two lines each of Xanthi WT, S/A, and RW grown in the dark for 7 days were determined. Figure $2 \mathrm{~B}$ shows that there were no significant differences in the hypocotyl length of these lines. This result was confirmed by the determination of instantaneous growth rates (data not shown). Taken together, these observations indicate that the effect is seen only when the rice phytochrome is activated by red light and is not simply the result of a nonspecific inhibitory effect caused by the expression of rice phyA itself.

To study the observed differences in sensitivity to red light in more detail, the relative hypocotyl length (percent hypocotyl length of dark-grown seedlings/ of seedling populations grown under different red light intensities was determined. Figure $3 \mathrm{~A}$ compares the short populations of the two transgenic lines S/A2 and RW2. For the S/A2 line, the inhibition of hypocotyl elongation was saturated at very low red light intensities $(1.2 \times$ $\left.10^{-3} \mathrm{~W} / \mathrm{m}^{2}\right)$, whereas the RW2 line reached this saturation level at a red light intensity $\left(3 \mathrm{~W} / \mathrm{m}^{2}\right)$ that was about three orders of magnitude higher. The threshold of the observed response is not altered, but the amplitude of the response observed in case of the S/A phyA-expressing seedlings is increased significantly. These results were confirmed with homozygous lines expressing RW and $\mathrm{S} / \mathrm{A}$ (data not shown). Figure $3 \mathrm{~B}$ shows the results for the corresponding long wild-type seedlings. These seedlings lost the transgene owing to segregation and were used as an internal control. Note that the long and short seedling population could not be distinguished at the lowest red light intensity used $\left(1.2 \times 10^{-3} \mathrm{~W} / \mathrm{m}^{2}\right)$. Therefore, the average of the relative hypocotyl length of the entire seedling population was included in the graph for the short (Fig. 3A) and the long population (Fig. 3B). The large difference in the relative hypocotyl length at the lowest red light intensity was the result of the transgenic S/A seedlings, which were more sensitive to red light than the transgenic RW seedlings. This was confirmed by experiments performed with seedling populations of homozygous S/A and RW lines (data not shown). The difference in the relative hypocotyl length between the long RW2 and S/A2 populations exposed to $1.2 \times 10^{-3}$ $\mathrm{W} / \mathrm{m}^{2}$ red light was an exception (Fig. 3D) and was not found in the analysis of the other lines.

The experiment described in Figure $3 \mathrm{~A}$ was carried out for six independent transgenic RW lines and four independent S/A lines. Figure $3 \mathrm{C}$ shows the average values of the analysis of the short seedling populations. This result demonstrates that the observations made for lines RW2 and S/A2 are not exceptional but hold true for all transgenic lines analyzed so far. Figure $3 \mathrm{D}$ summarizes the results obtained for the long wild-type seedling populations compared with untransformed tobacco var. Xanthi control seedlings. The standard deviation for the 
A

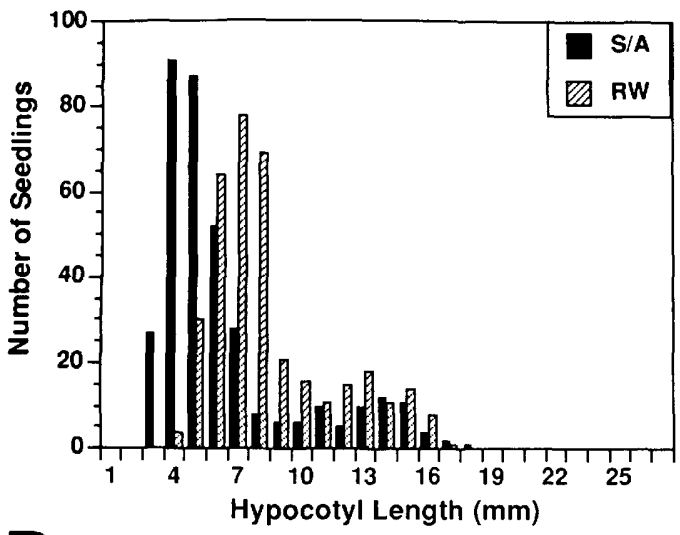

8

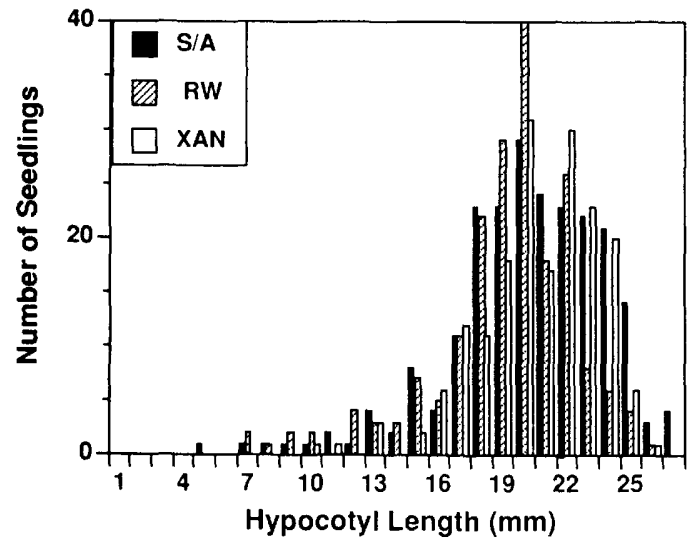

Figure 2. $(A)$ Comparison of hypocotyl length of $T_{1}$ seedlings of six tobacco lines expressing S/A phyA mutant (S/A lines) and six tobacco lines expressing RW phyA (RW lines). After sowing, the seedlings were exposed to white light for $24 \mathrm{hr}$ and grown under $15 \times 10^{-3} \mathrm{~W} / \mathrm{m}^{2}$ red light. The hypocotyl length of 60 seedlings of each line was determined 7 days after sowing. The diagram shows the number of seedlings vs. hypocotyl length. $(B)$ Comparison of hypocotyl length of $T_{1}$ seedlings of two independent Xanthi lines, two S/A lines, and two RW lines. After imbibition, the seedlings were grown for 7 days in darkness. The hypocotyl length of 60 seedlings was determined for each line. The diagram shows the number of seedlings vs. hypocotyl length.

long populations was generally higher than the standard deviation for the short seedling populations. One reason for this appears to be the varying vitality of the seedlings, which is largely determined by the amount of starch and other material stored in the seed. There was almost no variation in the results obtained with independent transgenic lines; in particular, the curves obtained for the short populations were virtually congruent.

Chlorophyll accumulation in seedlings is another physiological response that is known to be influenced by phytochrome. We found that the chlorophyll content increases with increasing red light intensity; however, there was no significant difference in the amount of accumulated chlorophyll between seedlings expressing S/A phyA and RW phyA and the untransformed tobacco controls at all light intensities examined (data not shown). This result contrasts with the high chlorophyll content of mature transgenic plants expressing rice phyA, as compared with untransformed control plants. The reason for this discrepancy is not clear at present and is being studied.

In the first section we have described the observation that compared with RW seedlings, S/A seedlings are characterized by a higher amplitude of response, in terms of inhibition of hypocotyl elongation by red light. The experiments described below were designed to determine whether (1) this observed increase in the amplitude was the result of changes in the physical properties of the S/A phytochrome, (2) these effects were caused by differences in expression levels and/or stability, and (3) there is a qualitative, functional difference between S/A and RW.

\section{Partial purification of $S / A$ phy $A$ and difference spectrum}

To test whether the chromophore is attached to the mutated phyA and whether the mutant phyA undergoes photoconversion, the S/A phyA was partially purified from extracts of dark-adapted leaves of transgenic tobacco plants by DEAE-Sepharose chromatography. Protein was eluted from the DEAE-Sepharose column by a linear $\mathrm{KCl}$ gradient (Fig. 4A). The protein and phytochrome content were determined spectrophotometrically. Individual fractions were concentrated by ammonium sulfate precipitation and analyzed for the presence of the S/A mutant phyA and for the endogenous tobacco phyA by immunoblotting (Kay et al. 1989b). The rice S/A phyA can be specifically detected by the monoclonal anti-rye antibody mAR14. The endogenous tobacco phyA was detected with mAP5, a monoclonal antibody specific for dicot phyA. Figure $4 \mathrm{~B}$ shows that the bulk of S/A phyA eluted in fractions 9 and 10, whereas the major fraction of tobacco phyA eluted in fractions 13 and 14 . The chromatographic separation of S/A phyA from endogenous tobacco phyA makes it possible to determine specifically the difference spectrum of S/A phyA. Figure $4 \mathrm{D}$ shows that the difference spectrum of $\mathrm{S} / \mathrm{A}$ phyA is very similar to that obtained for rice phyA extracted from etiolated rice seedlings (Fig. 4E). Moreover, the dark reversion of S/A phyA is not significantly faster than that of RW phyA measured in vitro (data not shown).

\section{Expression levels and stability of $S / A$ phyA}

We examined whether the observed changes in the red light response of S/A seedlings were simply caused by differences in the expression level. To this end, we measured steady-state rice phyA levels in independent S/A and RW lines by immunoblotting. As expected, the steady-state levels of RW phyA varied considerably among independent transgenic lines, probably owing to chromosomal position effects on the transgene expression. However, the levels were not significantly higher in the S/A lines as compared with the RW lines (Fig. $5 \mathrm{~A}, \mathrm{~B} \mid$. It is important to note that the expression level of 
Figure 3. Relative hypocotyl length (percent of the hypocotyl length of dark-grown seedlings) of seedling populations grown under different red light intensities. Seeds were imbibed under white light for $24 \mathrm{hr}$ and grown for 6 days either in darkness or under different red light intensities $\left(0.14 \times 10^{-3} \mathrm{~W} / \mathrm{m}^{2} ; 1.2 \times 10^{-3} \mathrm{~W} / \mathrm{m}^{2}\right.$; $\left.15 \times 10^{-3} \mathrm{~W} / \mathrm{m}^{2} ; 3000 \times 10^{-3} \mathrm{~W} / \mathrm{m}^{2}\right)$. The light intensities are given on the $x$ axis in logarithmic scale. The hypocotyl lengths of 60 seedlings were determined for each line and light intensity. Vertical bars indicate the standard deviation. $(A)$ Relative hypocotyl length of short (s) seedling populations of transgenic line S/A2 expressing the S/A phyA and the transgenic line RW2 expressing the RW phyA. $(B)$ Relative hypocotyl length of the long (1) seedling population of transgenic lines S/A2 and RW2 obtained from the same experiment as in $A$. These seedlings serve as internal controls. $(C)$ Average of the relative hypocotyl length of the short population of $T_{1}$ seedlings of four independent S/A lines and six independent RW lines. Seedlings were grown as described in $A$. (D) Average of the relative hypocotyl length of the long (1) seedling populations from S/A and RW lines, as well as three independent experiments carried out with untransformed tobacco var. Xanthi seedlings (XAN).

A

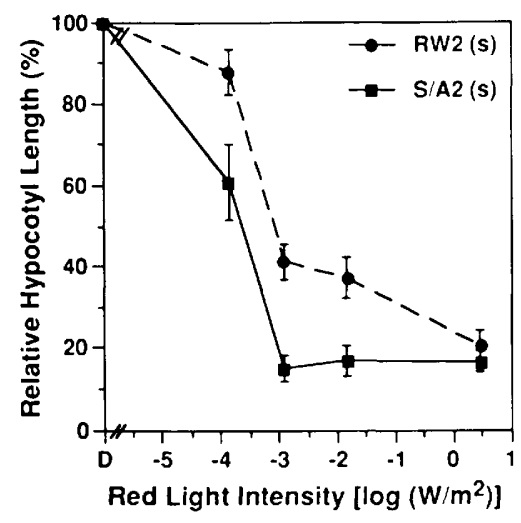

C

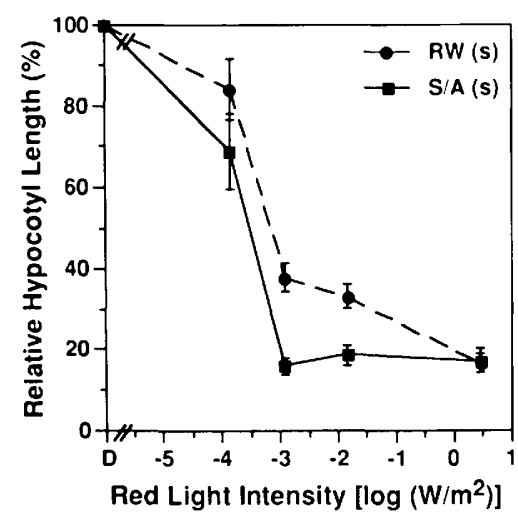

B

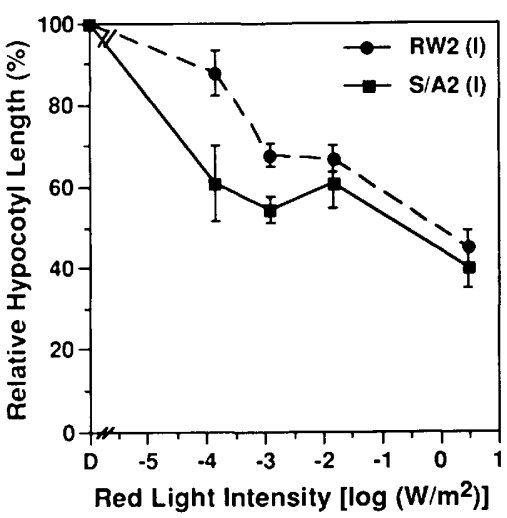

D

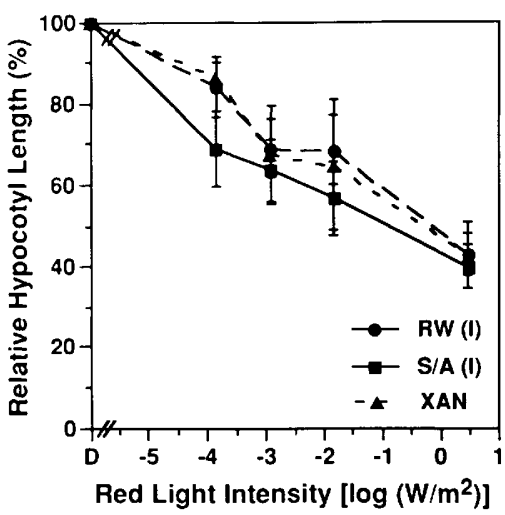

rice phyA in the RW2 line was much higher than in the $\mathrm{S} / \mathrm{A} 2$ line. The S/A line expressing the lowest level of S/A phyA (S/A2, Fig. 5A) showed the observed increase of the amplitude of the red light response even when compared with the RW line expressing the highest RW phyA level (RW2, Fig. 5B; cf. Fig. 3A). Generally, the steady-state level of expression did not affect the phenotypical differences between the S/A and RW lines described above.

We also examined whether RW and S/A phyA differ in stability. Because the Pfr form of phyA is degraded rapidly, a change in the stability of the Pfr form could cause the observed difference in red light response. This question was addressed by two experiments. The steady-state levels of S/A and RW phyA were determined in seedlings grown under the same conditions used for the hypocotyl elongation assays. At $1.2 \times 10^{-3} \mathrm{~W} / \mathrm{m}^{2}$ or higher red light intensities, the seedlings contained only low or undetectable levels of S/A and RW phyA (Fig. 6A,C). There were no major differences detectable between S/A and RW phyA levels under the same growth conditions in which the S/A lines exhibited shorter hypocotyls. As a control, the endogenous tobacco phyA pool was analyzed in parallel (Fig. 6B,D). The tobacco phyA could be detected in seedlings grown under $1.2 \times 10^{-3} \mathrm{~W} / \mathrm{m}^{2}$. This difference was probably the result of the higher affinity of the antibody mAP5 for dicot phyA as opposed to the lower affinity of the antibody mAR14 for monocot phyA.

We then compared the degradation of S/A and RW phyA. Seven-day old, dark-grown transgenic seedlings were exposed to red light $\left(15 \times 10^{-3} \mathrm{~W} / \mathrm{m}^{2}\right)$ for $1-24 \mathrm{hr}$. Immunoblots were probed with antibodies specific for monocot or dicot phyA. Upon transfer to red light, the rice phyA pool depleted very rapidly. S/A phyA (Fig. 7A) did not seem to be significantly more stable than RW phyA (Fig. 7C). As a control, duplicates of these blots were stained with antibodies specific for dicot phyA (Fig. 7B,D). Rice phyA appeared to be more stable than endogenous tobacco phyA under these conditions.

\section{Discussion}

In this paper we show that substitution of the first 10 serine residues by alanine residues at the amino terminus of rice phyA does not abolish the photoreceptor function. Interestingly, the mutated phytochrome appears to have a higher biological activity when compared with wild-type rice phyA. The amplitude of the red light response with respect to the inhibition of hypocotyl elongation is increased in S/A seedlings as compared with seedlings expressing RW phyA. In addition, it is remarkable that the threshold of this response is apparently unchanged (Fig. 3). Because the hypocotyl length of 

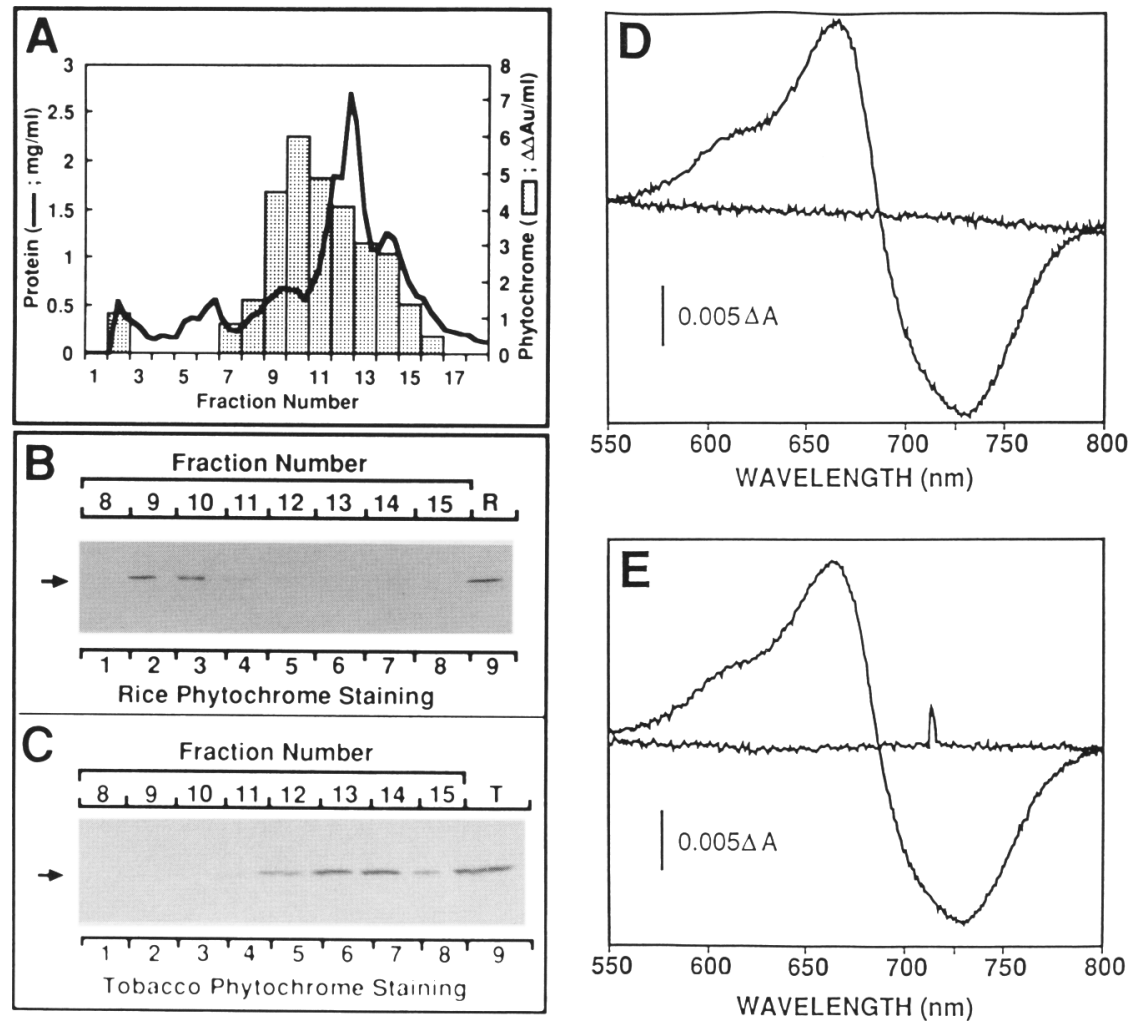

Figure 4. (A) Protein and phytochrome elution profile of DEAE-Sepharose chromatography of extracts from dark-adapted tobacco leaves of transgenic line S/A2. Extracts obtained from 80 grams of leaves were fractionated as described in Materials and methods. Fractions were assayed for protein content and phytochrome by spectrophotometry. $(B)$ Immunoblot analysis of DEAE fractions of S/A2 extracts. Detection of the S/A phyA mutant protein /staining with monocot-specific monoclonal antibody mAR14). (Lanes 1-8) 1/100 aliquot of DEAE fractions 8-15, respectively; (lane 9) extract of dark-adapted rice seedlings. The arrow indicates the position of the $116-\mathrm{kD}$ marker ( $\beta$-galactosidase). $(C)$ Detection of the tobacco phyA protein (stained with dicot-specific monoclonal antibody mAP5). (Lanes 1-8) 1/100 aliquot of DEAE fractions 8-15, respectively; (lane 9) extract of dark-adapted tobacco leaves. The arrow indicates the position of the $116-\mathrm{kD}$ marker. (D) Difference spectrum ( $\mathrm{Pr}$ minus Pfr) of partially purified S/A phyA mutant. Fractions 9 and 10 eluted from the DEAE-Sepharose column $(B)$ was concentrated by ammonium sulfate precipitation. $(E)$ Difference spectrum of rice phyA extracted from etiolated rice seedlings. etiolated seedlings does not differ significantly between wild-type Xanthi and transgenic S/A and RW lines (Fig. $2 \mathrm{~A}$, we conclude that the phenotypic difference is neither caused by changes in the kinetics of the germination process nor by general inhibitory effects of S/A phyA. We found that the observed phenotypic effect is not correlated with the steady-state expression levels of the transgenes. Under continuous red light irradiation of $1.2 \times 10^{-3} \mathrm{~W} / \mathrm{m}^{2}$ and higher, $\mathrm{S} / \mathrm{A}$ and $\mathrm{RW}$ phyA amounts drop below the level of detection of the Western blot technique used. This steep drop in rice phyA content, which is caused by the rapid degradation of phyA in the Pfr form, is correlated with a significant inhibition of hypocotyl elongation. These results suggest that a low concentration of rice phyA, which is constitutively resynthesized because the gene is under $35 \mathrm{~S}$ promoter control, is sufficient to induce the observed increase in the amplitude of the red light response.

We investigated whether S/A phyA has an increased stability that could lead to an increase in the steady-state S/A phyA level in comparison with RW phyA in seedlings grown under different light conditions for 6 days (Fig. 6). However, we found that the amounts of S/A and RW phyA are below detection levels, thus making it impossible to compare phyA amounts under these experimental conditions. Analysis of their degradation revealed that the Pfr form of S/A phyA appears not to be much more stable than the Pfr form of RW phyA. Taken together, our results indicate that the increase in the amplitude of the red light response is independent of the

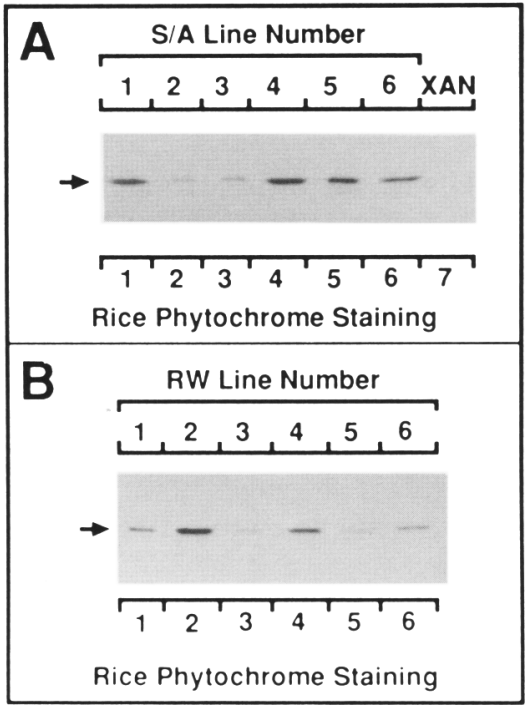

Figure 5. (A) Western blot analysis of six independent S/A tobacco lines expressing the rice phyA mutant (stained with mAR14). (Lanes 1-6) Extracts of S/A lines 1-6, (XAN) extract of untransformed tobacco leaves var. Xanthi. Each lane contains $20 \mu \mathrm{g}$ of protein extracted from young leaves of dark-adapted 17 days) tobacco plantlets grown in tissue culture. $(B)$ Western blot analysis of six independent RW tobacco lines expressing rice wild-type phyA (stained with mAR14). (Lanes 1-6) Extracts of RW lines 1-6. Each lane contains $20 \mu \mathrm{g}$ of protein extracted from young leaves of dark-adapted tobacco plants (cf. $A$ ). 


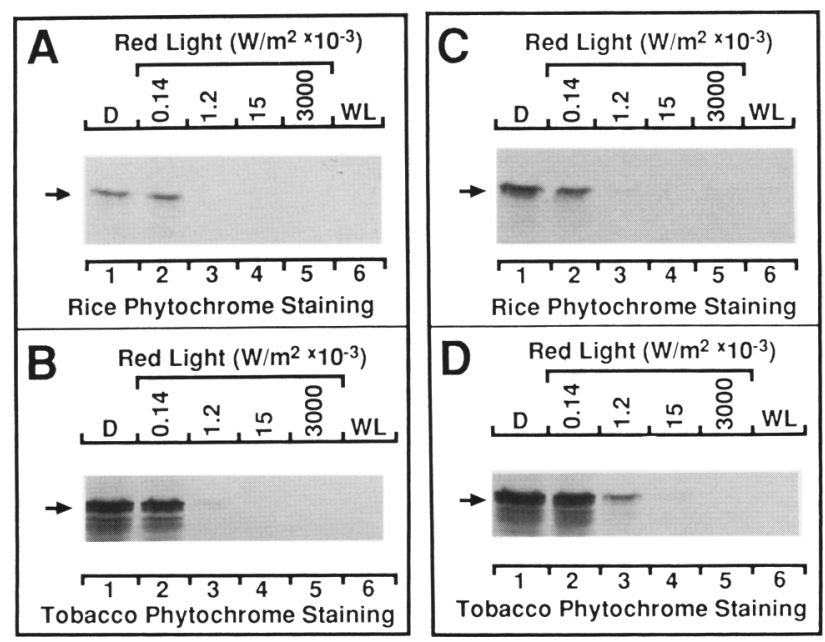

Figure 6. Immunoblot analysis of phytochrome in extracts of 7-day-old seedlings grown under different light conditions. $(A)$ Detection of the S/A phyA mutant protein in extracts of S/A2 seedlings (staining with $\mathrm{MAR} 14$ ). (Lane 1) Extract of S/A2 seedlings grown in the dark; (lanes 2-5) extracts of seedlings grown under different red light intensities. (Lane 2) $0.14 \times 10^{-3}$ $\mathrm{W} / \mathrm{m}^{2}$; (lane 3) $1.2 \times 10^{-3} \mathrm{~W} / \mathrm{m}^{2}$; (lane 4) $15 \times 10^{-3} \mathrm{~W} / \mathrm{m}^{2}$; (lane 5) $3000 \times 10^{-3} \mathrm{~W} / \mathrm{m}^{2}$; (lane 6) seedlings grown under continuous white light. In each lane, $40 \mu \mathrm{g}$ of protein was loaded. The arrow indicates the position of the $116-\mathrm{kD}$ marker $(\beta-\mathrm{ga}-$ lactosidase). $(B)$ Duplicate of Western blot described in $A$, stained with mAP5 to detect the endogenous tobacco phyA protein. $(C)$ Detection of RW phyA protein in extracts of RW1 seedlings grown under different light regimes/staining with mAR14). (For descriptions of the different lanes, see description of $A$, above.) $(D)$ Duplicate of Western blot described in $C$ (above) stained with mAP5 to detect the endogenous tobacco phyA protein.

expression level and cannot be attributed to a major difference in the stability of the Pfr form. This implies that there is a qualitative difference between S/A and RW phyA. Consistent with this interpretation, we found that the observed phenotypic differences are independent of the absolute expression level in independent transgenic lines.

The serine-to-alanine substitution in the amino-terminal domain of phyA is expected to alter the secondary structure and hydrophobic moment of this region of the molecule. There is suggestive evidence that the aminoterminal domain of phytochrome undergoes significant conformational changes upon photoconversion from the Pr to the Pfr form (Vierstra and Quail 1986; Yamamoto 1990; Parker et al. 1991). The proteolytic removal of the amino-terminal 6-10 kD leads to a shift in the absorption maximum of Pfr from 730 to $722 \mathrm{~nm}$ (Vierstra and Quail 1983), indicating that this amino-terminal domain interacts with the chromophore environment when phytochrome is in the Pfr form. We found that the S/A mutant not only exhibits photoreversibility, but its difference spectrum is very similar to that of the phytochrome extracted from etiolated rice seedlings. These results, as well as the observed low rate of dark reversion, indicate that the mutations do not seem to cause any major changes in the spectral properties of this molecule. There is some evidence that the amount of $\alpha$-helical content in the amino-terminal domain increases upon photoconversion to the Pfr form (Chai et al. 1987; Vierstra et al. 1987). The serine-to-alanine substitutions may extend the predicted $\alpha$-helix toward the amino terminus of the molecule, thereby facilitating and/or stabilizing the conformational changes following photoconversion. Besides these possible structural changes, the amino acid substitutions also increase the hydrophobicity of the amino-terminal domain, which might lead to the observed increase in biological activity.

The observed increase in the biological activity of S/A phyA might also be caused by the removal of potential phosphorylation sites. Phytochrome is known to be a phosphoprotein (Quail et al. 1978; Hunt and Pratt 1980), and more recently, a polycation-stimulated kinase activity has been found to be associated with purified phytochrome (Wong et al. 1986). Although the phosphorylation site of this kinase has been mapped to the aminoterminal serine-rich region (McMichael and Lagarias 1990), whether these serine residues are phosphorylated in vivo and the functional implications of this phosphorylation are not clear.

The results described here demonstrate that serine res-
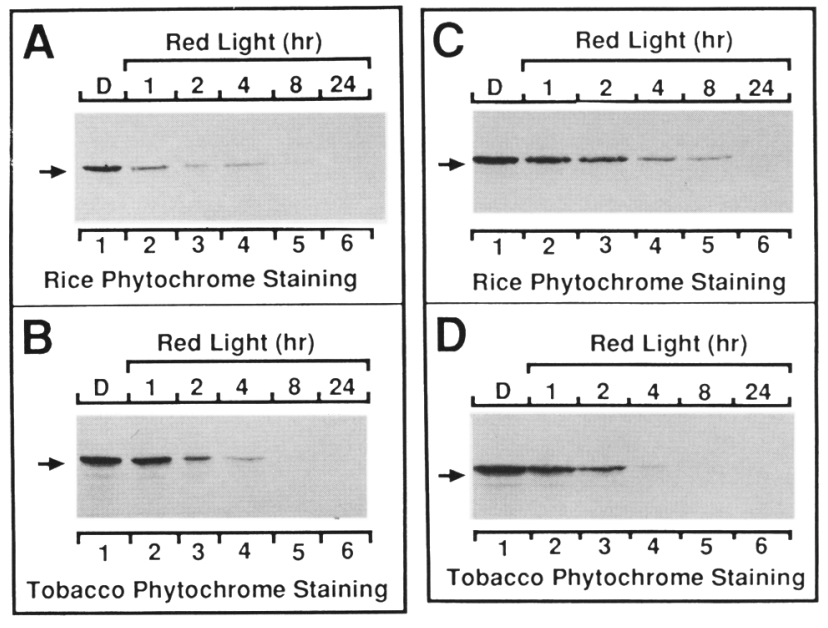

Figure 7. Immunoblot analysis of phyA in extracts of 6-day-old dark-adapted tobacco seedlings subjected to different periods of red light illumination (red light intensity, $15 \times 10^{-3} \mathrm{~W} / \mathrm{m}^{2}$ ). $(A)$ Detection of rice phyA mutant in extracts of S/A2 seedlings (staining with mAR14). (Lane 1) Extract of S/A2 seedlings grown in darkness for 7 days; (lane 2) subsequently irradiated with red light for $1 \mathrm{hr}$; (lane 3) $2 \mathrm{hr}$; (lane 4) $4 \mathrm{hr}$; (lane 5) $8 \mathrm{hr}$; (lane 6) $24 \mathrm{hr}$. In each lane, $40 \mu \mathrm{g}$ of protein was loaded. The arrow indicates the position of the $116-\mathrm{kD}$ marker ( $\beta$-galactosidase). $(B)$ Duplicate of Western blot described for $A$ (above), stained with mAP5 to detect the endogenous tobacco phyA. $(C)$ Detection of rice phyA protein in extracts of RWl seedlings (staining with mAR 14). (For description of the various lanes, see $A$, above.) (D) Duplicate of Western blot described for $C$ (above), stained with mAP5 for the detection of the endogenous tobacco phyA protein. 
idues at positions $2-4,10-14,19$, and 20 are not necessary for the function of phyA, because substitution of these serine residues by alanine does not abolish but, rather, increases the biological activity of S/A phyA. An attractive possibility is that serine phosphorylation in the amino-terminal region is involved in the desensitization of the phytochrome signaling system. After photoconversion, Pfr could be inactivated by phosphorylation before entering the degradation pathway. According to this model, the observed increase in biological activity seen in S/A phyA is the result of a lack of, or a decrease in, desensitization, resulting in a prolongation of the "active" state of the Pfr form. Although no major differences were observed between S/A phyA and wild-type phyA in the degradation kinetics of the bulk phytochrome pool, the active fraction may only constitute a small proportion of the total Pfr pool (Fig. 7). Alternatively, the increase in the half-life of the active Pfr state could be too small to be detected by our experiments.

In this report we have used a transgenic plant system to assay for the biological activity of a phyA mutant. Because of the higher activity of the S/A phyA mutant, alterations of the phyA molecule could be made in the context of this mutant that can be used as a pseudo-wildtype. This should increase the sensitivity of the assay for future structure/function studies of the phyA molecule.

\section{Materials and methods}

\section{Constructs and tobacco transformation}

The first 10 serine codons in the amino-terminal domain of rice phy $A$ were changed to alanine codons using polymerase chain reaction (PCR)-mediated mutagenesis (Fig. 1B). The mutations were introduced into the $3^{\prime}$ oligonucleotide used for PCR amplification. The $3^{\prime}$ oligonucleotide covers the region of a unique Smal site in phyA. The amplified PCR fragments were subcloned into pUC19 and sequenced. The mutated fragment was inserted into the rice phyA cDNA as an EcoRI-SmaI fragment, and the reconstituted cDNA clone encoding the serine-to-alanine substitution was cloned into the expression casette pMON530 (Cuozzo et al. 1987) containing the 35S promoter and the polyadenylation signal of the $r b c \mathrm{~S}$-E9 gene from the pea. As a control, the wild-type rice phy $A$ cDNA (Kay et al. 1989b) was inserted into the same vector. Agrobacterium tumefaciens (strain GV3111SE) cells harboring the described plasmid vectors were used to inoculate leaf discs of $N$. tabacum cv. Xanthi and regenerated shoots were selected on kanamycin. $T_{0}$ plants were selfed, and $T_{1}$ seeds were used for the experiments described. For phytochrome extractions, plants were grown in soil in a growth cabinet (Koitotron KG-206HL-D, Koito, Tokyo, Japan) at $25^{\circ} \mathrm{C}$ under continuous illumination.

\section{Hypocotyl elongation assay}

For hypocotyl length determinations, seeds were germinated on $0.8 \%$ agar medium containing $0.1 \times$ Murashige and Skoog salt mixture. The seeds were imbibed under continuous white light $\left(6.0 \mathrm{~W} / \mathrm{m}^{2}\right)$ for $24 \mathrm{hr}$ to induce seed germination and then subjected to the various light treatments for 6 days before hypocotyl lengths were measured at the end of the light treatment.

\section{Chlorophyll accumulation}

Seedlings were grown under different intensities of red light $\left(0.14 \times 10^{-3} \mathrm{~W} / \mathrm{m}^{2} ; 1.2 \times 10^{-3} \mathrm{~W} / \mathrm{m}^{2} ; 15 \times 10^{-3} \mathrm{~W} / \mathrm{m}^{2} ;\right.$ $3000 \times 10^{-3} \mathrm{~W} / \mathrm{m}^{2}$ ) for 6 days. After this period the seedlings were transferred to white light $\left(6.0 \mathrm{~W} / \mathrm{m}^{2}\right)$ for $3 \mathrm{hr}$. Two samples of 20 seedlings each (this experiment was done twice) were transferred into $1.5-\mathrm{ml}$ reaction tubes containing $1 \mathrm{ml}$ of dimethylformamide and kept in darkness at $4^{\circ} \mathrm{C}$ for $24 \mathrm{hr}$. The tubes were mixed on a reaction tube mixer (Eppendorf) for 30 min and subsequently centrifuged at $10,000 \mathrm{rpm}$ for $10 \mathrm{~min}$. Different dilutions of the clear supernatant were used for chlorophyll determinations (Moran and Porath 1980) with a fluorescence spectrophotometer (model F-3010, Hitachi, Ltd., Tokyo, Japan).

\section{Phytochrome extraction and analysis}

Procedures for extraction, partial purification, and detection of phytochrome were essentially performed as described (Nagatani et al. 1989). Phytochrome was partially purified from leaves of dark-adapted (3-5 days) transgenic tobacco plants by DEAESepharose chromatography. Immunoblots were probed with the anti-rye phyA monoclonal antibody, mAR14 (Kay et al. 1989b), and the anti-pea phyA monoclonal antibody, mAP5 (Nagatani et al. 1984). The extraction procedure for the Western blot analysis was modified as follows. Pieces of dark-adapted tobacco tissue were illuminated on ice with red light for $5 \mathrm{~min}$ and frozen immediately in liquid nitrogen. The tissue was homogenized in an extraction buffer $10.1 \mathrm{M}$ phosphate buffer at $\mathrm{pH} 7.8$; 2 mM EDTA; $1 \mathrm{mM}$ PMSF; $10 \mathrm{~mm}$ iodoacetamide; $56 \mathrm{~mm} \beta$-mercaptoethanol). The extract was centrifuged in a refrigerated tabletop centrifuge at $15,000 \mathrm{rpm}$ for $10 \mathrm{~min}$. The supernatant was discarded and the sediment was extracted with loading buffer. Using this procedure, phytochrome was quantitatively recovered from the pellet fraction. Protein concentration in individual samples was determined using the Bradford assay (Bio-Rad) and confirmed by Coomassie staining of SDS protein gels. Difference spectra of phytochrome were measured with a recording spectrophotometer (model 3410, Hitachi, Ltd., Tokyo, Japan). One $\Delta(\Delta \mathrm{A})$ unit was defined as the amount of phytochrome that shows 0.001 photoreversible absorbance difference change between 660 and $730 \mathrm{~nm}$ when dissolved in $1 \mathrm{ml}$ and measured in a $10-\mathrm{mm}$ path length cuvette.

\section{Light sources}

White fluorescent tubes (20W, Fl20SS-W/18, Toshiba, Tokyo, Japan) with an intensity of $6.0 \mathrm{~W} / \mathrm{m}^{2}$ were used for the induction of germination and for in vitro culture of tobacco plants. Red light $\left(0.4-3.0 \mathrm{~W} / \mathrm{m}^{2}\right)$ was obtained from the same light source filtered through one layer of acrylic $13 \mathrm{~mm}$, red, Shinkolite A102, Mitsubishi, Tokyo, Japan). The different red light intensities were obtained by combinations of one red filter /described above) and different gray filters (vinyl resin, darker gray, 3-mm Takiron plate S-909; vinyl resin, lighter gray, 2-mm Takiron plate S-802; Takiron Co., Tokyo, Japan). Light intensities were determined with a radiometer.

\section{Acknowledgments}

We are very grateful to Ellen Leheny, Ling Ren, Stan Sotnikov, and K. Fujiwara for expert technical assistance, to Arnold Hinton for photography, and to Wendy Roine for typing. We thank the members of the Laboratory of Plant Molecular Biology, the Rockefeller University, and the members of the Frontier Research Program RIKEN, as well as Professor Eberhard Schäfer, for many helpful discussions and for comments on the manu- 
script. J.S. was supported by a grant from the Deutsche Forschungsgemeinschaft. This work was supported by grants from the Rockefeller Foundation and the Human Frontier Science Program.

The publication costs of this article were defrayed in part by payment of page charges. This article must therefore be hereby marked "advertisement" in accordance with 18 USC section 1734 solely to indicate this fact.

\section{References}

Boylan, M.T. and P.H. Quail. 1989. Oat phytochrome is biologically active in transgenic tomatoes. Plant Cell 1: 765-773.

- 1991. Phytochrome A overexpression inhibits hypocotyl elongation in transgenic Arabidopsis. Proc. Natl. Acad. Sci. 88: 10806-10810.

Chai, Y.-G., P.-S. Song, M.-M. Cordonnier, and L.H. Pratt. 1987. A photoreversible circular dichroism spectral change in oat phytochrome is suppressed by a monoclonal antibody that binds near its $\mathrm{N}$-terminus and by chromophore modification. Biochemistry 26: 4947-4952.

Christensen, A.H. and P.H. Quail. 1989. Structure and expression of a maize phytochrome-encoding gene. Gene 85: 381390.

Cordonnier, M.M., H. Greppin, and L.H. Pratt. 1985. Monoclonal antibodies with differing affinities to the red-absorbing and far-red-absorbing forms of phytochrome. Biochemistry 24: 3246-3253.

Cuozzo, M., K. O'Connel, W. Kaniewski, R.-X. Fang, and N.-H. Chua. 1987. Viral protection in transgenic plants expressing the cucumber mosaic virus coat protein or its antisense RNA. Biotechnology 6: 549-557.

Furuya, M. 1987. Phytochrome and photoregulation in plants. Academic Press, Tokyo, Japan.

- 1989. Molecular properties and biogenesis of phytochrome I and II. Adv. Biophys. 25: 133-167.

Hershey, H.P., R.F. Barker, K.B. Idler, J.L. Lissemore, and P.H. Quail. 1985. Analysis of cloned cDNA and genomic sequences for phytochrome: Complete amino acid sequence for two gene products expressed in etiolated Avena. Nucleic Acids Res. 13: 8543-8559.

Hunt, R.E. and L.H. Pratt. 1980. Partial characterization of undegraded oat phytochrome. Biochemistry 19: 390-394.

Jones, A.M., R.D. Vierstra, S.M. Daniels, and P. Quail. 1985. The role of separate molecular domains in the structure of phytochrome from etiolated Avena sativa L. Planta 164: 501-506.

Kay, S.A., B. Keith, K. Shinozaki, and N.-H. Chua. 1989a. The sequence of the rice phytochrome gene. Nucleic Acids Res. 17: 2865-2866.

Kay, S.A., A. Nagatani, B. Keith, M. Deak, M. Furuya, and N.-H. Chua. $1989 \mathrm{~b}$. Rice phytochrome is biologically active in transgenic tobacco. Plant Cell 1: 775-782.

Keller, J.M., J. Shanklin, R.D. Vierstra, and H.P. Hershey. 1989. Expression of a functional monocotyledonous phytochrome in transgenic tobacco. EMBO J. 8: 1005-1012.

Kendrick, R.E. and G.H.M. Kronenberg. 1986. Photomorphogenesis in plants. Martinus Nijhoff/Dr. W. Junk Publishers, Dordrecht, The Netherlands.

Lagarias, J.C. and F.M. Mercurio. 1985. Structure and function studies on phytochrome. I. Biol. Chem. 260: 2415-2423.

Lagarias, J.C. and H. Rapoport. 1980. Chromopeptides from phytochrome. The structure and linkage of the Pr form of the phytochrome chromophore. I. Am. Chem. Soc. 102: 48214828.
McMichael, R.W. Jr. and J.C. Lagarias. 1990. Phosphopeptide mapping of Avena phytochrome phosphorylated by protein kinases in vitro. Biochemistry 29: 3872-3878.

Moran, R. and D. Porath. 1980. Chlorophyll determination in intact tissue using $\mathrm{N}, \mathrm{N}$-dimethylformamide. Plant Physiol. 65: $478-479$.

Nagatani, A., K. Yamamoto, M. Furuya, T. Fukumoto, and A. Yamashita. 1984. Production and characterization of monoclonal antibodies which distinguish different surface structures of pea phytochrome. Plant Cell Physiol. 25: 10591068.

Nagatani, A., R.E. Kendrick, M. Koornneef, and M. Furuya. 1989. Partial characterization of phytochrome I and II in etiolated and de-etiolated tissues of a photomorphogenetic mutant (lh) of cucumber (Cucumis sativus L.) and its wild type. Plant Cell Physiol. 30: 685-690.

Nagatani, A., S.A. Kay, M. Deak, N.-H. Chua, and M. Furuya. 1991. Rice type I phytochrome regulates hypocotyl elongation in transgenic tobacco seedlings. Proc. Natl. Acad. Sci. 88: 5207-5211.

Parker, W., M. Romanowski, and P.-S. Song. 1991. Conformation and its functional implications in phytochrome. In Phytochrome properties and biological action (ed. B. Thomas and C.B. Johnson), pp. 85-112. Springer-Verlag, Berlin/ Heidelberg, Germany.

Quail, P.H. 1991. Phytochrome: A light-activated molecular switch that regulates plant gene expression. Annu. Rev. Genet. 25: 389-409.

Quail, P.H., W.R. Briggs, and L.H. Pratt. 1978. In vivo phosphorylation of phytochrome. In Carnegie Institution Annual Report, pp. 342-344. Carnegie Institution of Washington, Stanford, CA.

Sato, N. 1988. Nucleotide sequence and expression of the phytochrome gene in Pisum sativum: Differential regulation by light of multiple transcripts. Plant Mol. Biol. 11: 697-710.

Sharrock, R.A. and P.H. Quail. 1989. Novel phytochrome sequences in Arabidopsis thaliana: Structure, evolution, and differential expression of a plant regulatory photoreceptor family. Genes \& Dev. 3: 1745-1757.

Sharrock, R.A., J.L. Lissemore, and P.H. Quail. 1986. Nucleotide and amino acid sequence of a Curcurbita phytochrome cDNA clone: Identification of conserved features by comparison with Avena phytochrome. Gene 47: 287-295.

Smith, H. and G.C. Whitelam. 1990. Phytochrome, a family of photoreceptors with multiple physiological roles. Plant Cell Environ. 13: 695-707.

Thomas, B. and C.B. Johnson. 1991. Phytochrome properties and biological action. Springer-Verlag, Berlin, Germany.

Vierstra, R.D. and P.H. Quail. 1983. Photochemistry of 124 kilodalton Avena phytochrome in vitro. Plant Physiol. 72: 264-267.

- 1986. The protein. In Photomorphogenesis in plants (ed. R.E. Kendrick and G.H.M. Kronenberg), pp. 35-60. Marinus Nijhoff/Dr. W. Junk Publishers, Dordrecht, Holland.

Vierstra, R.D., P.H. Quail, T.-R. Hahn, and P.-S. Song. 1987. Comparison of the protein conformations between different forms (Pr and Pfr) of native (124 kD) and degraded (118/114 kD) phytochrome from Avena sativa. Photochem. Photobiol. 45: 429-432.

Wong, Y.-S., H.-C. Cheng, D.A. Walsh, and J.C. Lagarias. 1986. Phosphorylation of Avena phytochrome in vitro as a probe of light-induced conformational changes. I. Biol. Chem. 261: 12089-12097.

Yamamoto, K.T. 1990. Photoreversible absorption change and domain structure of phytochrome. Bot. Mag. Tokyo 103: 469-491. 


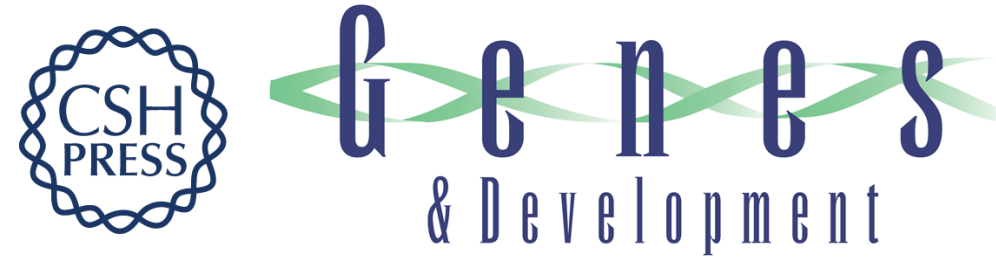

\section{Serine-to-alanine substitutions at the amino-terminal region of phytochrome $A$ result in an increase in biological activity.}

J Stockhaus, A Nagatani, U Halfter, et al.

Genes Dev. 1992, 6:

Access the most recent version at doi:10.1101/gad.6.12a.2364

References This article cites 29 articles, 9 of which can be accessed free at:

http://genesdev.cshlp.org/content/6/12a/2364.full.html\#ref-list-1

License

Email Alerting

Service

Receive free email alerts when new articles cite this article - sign up in the box at the top right corner of the article or click here.

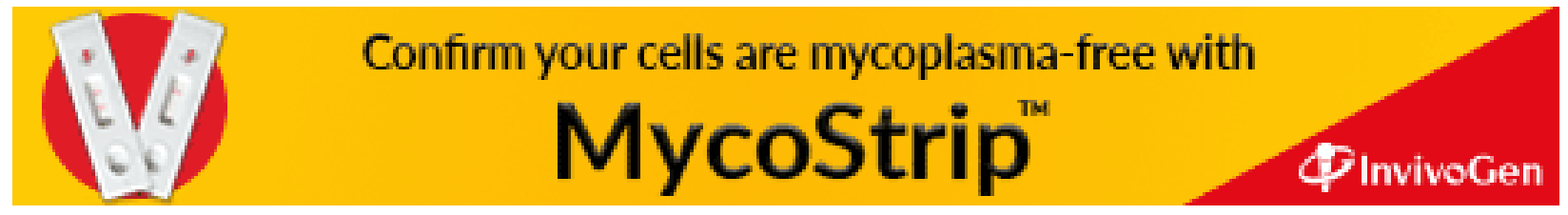

